\title{
Far-Infrared Few-Cycle-Pulse Generation in Quantum-Well Heterostructures under Femtosecond Laser Pumping
}

\author{
A.A. Belyanin ${ }^{a, b}$, V.V. Kocharovsky ${ }^{a, b}$, \\ Vl.V. Kocharovsky ${ }^{b}$, D.S. Pestov ${ }^{a, b}$ And M.O. Scully ${ }^{a, c, d}$ \\ ${ }^{a}$ Institute for Quantum Studies and Physics Department, Texas A\&M University \\ College Station, TX 77843, USA \\ ${ }^{b}$ Institute of Applied Physics, Russian Academy of Sciences \\ 46 Ulyanov str., 603950, Nizhny Novgorod, Russia \\ ${ }^{c}$ Department of Electrical Engineering, Texas A\&M University \\ College Station, TX 77843, USA \\ ${ }^{d}$ Departments of Chemistry, Aerospace \& Mechanical Engineering \\ Princeton University, Princeton, USA \\ It is shown that the resonant nonlinear-wave mixing in the semiconduc- \\ tor quantum wells under a femtosecond optical pulse excitation can be used \\ for the generation of the utmost short mid/far-infrared pulses with a few- or \\ even single-cycle duration defined by pump duration. \\ PACS numbers: 42.55.Px, 42.60.--v
}

\section{Introduction}

Different optoelectronic techniques, utilizing ultrashort optical laser pulses, to generate $\mathrm{THz}$ few-cycle pulses were suggested and experimentally realized in 90's; see, e.g., [1-9]. Also, the ultrafast coherence relaxation dynamics in heterostructures was investigated, including the charge oscillations in quantum wells (QWs) [10-14] and the Bloch oscillations in superlattices [15-17].

In the present paper we address the resonant nonlinear-wave mixing in the multiple-QW heterostructures under femtosecond laser pulse excitation as a potential source of the utmost short, i.e. few-cycle or single-cycle, mid/far-infrared pulses. Recently we suggested the use of the resonant nonlinear-wave mixing inside a semiconductor laser cavity as a basis for the new kind of continuous-wave room temperature mid/far-infrared lasers [18-20]. There was also an observation of the 10 - to 20 -cycle, i.e. $0.2-0.4 \mathrm{ps}$, mid-infrared pulses resulting from coherent 
charge oscillations (resonant mixing) in multiple QWs under femtosecond pulse excitation [21].

In Sec. 2 we analyze emission of the mid/far-infrared pulses for the planar multiple-QW structure optically pumped at the Brewster angle. In Sec. 3, in the frame of a simple three-level model of QWs, the mid/far-infrared coherence excitation is described analytically. Numerical results which include also a finite dephasing time are presented and compared with the analytical ones. In Sec. 4 we estimate the power of the mid/far-infrared radiation for typical GaAs-based heterostructures and give conclusions.

\section{Generation of mid/far-infrared radiation}

The optical femtosecond pulse passing through the active medium (multiple-QW structure) initiates difference-frequency mixing process due to the second-order electron (hole) nonlinearity $\chi^{(2)}$ and creates a resonant mid/far-infrared wave of nonlinear polarization with the $\boldsymbol{k}$-vector in the plane of QWs. If the optical pump pulse is TM-polarized and sent under the Brewster angle $\left(\tan \theta_{0}=\sqrt{\varepsilon}\right)$ to the surface of the heterostructure, in order to avoid reflection (see Fig. 1) the

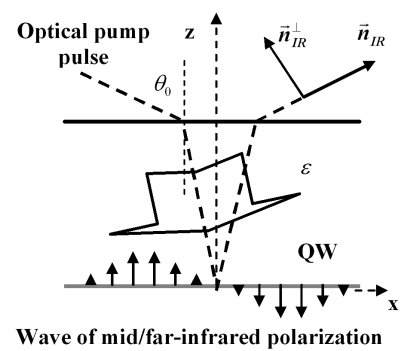

Fig. 1. Geometry of the coherence excitation and emission of the mid/far-infrared radiation.

generated mid/far-infrared field outside the structure should be found from

$$
\boldsymbol{E}_{\mathrm{IR}}(\boldsymbol{r}, t) \approx-\boldsymbol{n}_{\mathrm{IR}}^{\perp} \frac{2 \pi N_{0} N_{\mathrm{QW}}}{c \varepsilon^{3 / 2}} \frac{\partial}{\partial t} p\left(t-\frac{\boldsymbol{n}_{\mathrm{IR}} \boldsymbol{r}}{c}\right) .
$$

Here $p(t)$ is a dipole moment created by one electron on the intersubband transition, $N_{0}$ is the density of the dipoles per unit area of a $\mathrm{QW}, N_{\mathrm{QW}}$ is the number of QWs, $c$ is the speed of light in the vacuum, $\boldsymbol{n}_{\mathrm{IR}}$ is a unit vector in the direction of the mid/far-infrared wave propagation, $\boldsymbol{n}_{\mathrm{IR}}^{\perp}$ is a unit vector that lies in the plane of incidence, perpendicular to the propagation direction, and has a positive projection on $z$-axis, $\varepsilon$ is the dielectric constant of the material. For the sake of simplicity, dispersion of semiconductor materials is neglected, which is reasonable if the distance from QWs to the semiconductor surface is much less than the mid/far-infrared wavelength $\lambda_{\text {IR }}$. 
In the case of $z$-polarized mid/far-infrared transitions typical of the electron subbands in asymmetrical QWs, the mid/far-infrared signal vanishes for normal incidence of the pump pulse. Due to mixing of heavy and light holes in the valence band, the dipole moments of the valence-band intersubband transitions may be polarized in the QW-plane [22]; in this case the maximum of the mid/far-infrared signal will take place for the normal incidence of the pump pulse.

\section{Intraband coherence}

A simple three-level scheme of electron-hole states in QWs accounts well for the coherent quantum beats owing to the resonant difference-frequency mixing within the band width of the pump pulse. A schematic diagram is shown in Fig. 2. For typical GaAs/AlGaAs or InGaAs/GaAs structures, the frequencies of the interband transitions, $\omega_{21}$ and $\omega_{31}$, lie in the near-infrared (optical) region, $\lambda \approx 0.8-1 \mu \mathrm{m}$, whereas the frequency of the intersubband transition, $\omega_{32}$, can be varied in a wide range - from mid-infrared to far-infrared, $\lambda_{\mathrm{IR}} \approx 5-100 \mu \mathrm{m}$, and to the $\mathrm{THz}$ region. In symmetrical QWs, all three transitions may be dipole allowed due to mixing of heavy and light holes [20, 22].

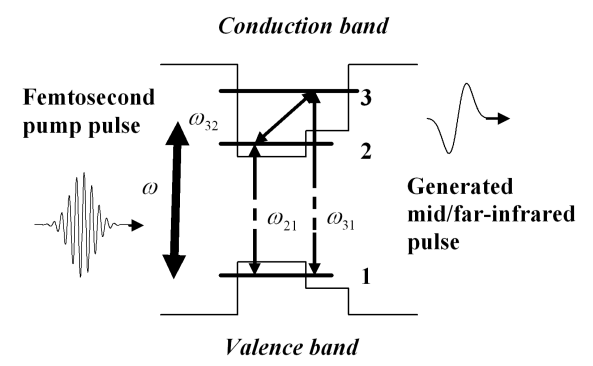

Fig. 2. A schematic diagram of the three-level system with two electron and one hole levels. Here $\omega_{21}$ and $\omega_{31}$ are the frequencies of the two dipole allowed interband transitions, $\omega$ - the frequency of the pump pulse, $\omega_{32}$ - the frequency of the dipole allowed intersubband transition.

An essential point of the schematic given is that the mean frequency of the pump pulse, $\omega$, is close to the frequencies of the interband transitions, $\omega_{21}$ and $\omega_{31}$, and the width of the pump pulse spectrum is large enough to span the difference between them, $\omega_{32}$.

In the simplest case of interest, when $\omega=\left(\omega_{21}+\omega_{31}\right) / 2$, the dipole moments $d_{21}$ and $d_{31}$ of the transitions $2-1$ and $3-1$ are set to be real and equal to each other (so that the Rabi frequencies are equal and real, $\Omega_{21}(t)=\Omega_{31}(t)=\Omega(t)=$ $d E(t) / \hbar)$, and a population transfer because of nonradiative processes is negligible (so that there are only two finite nonradiative constants, $\gamma_{32}$ and $\gamma_{21}=\gamma_{31}=\gamma$ ), equations for the density matrix,

$$
\rho_{21}(t)=\sigma_{21}(t) \mathrm{e}^{-\mathrm{i} \omega t}, \rho_{31}(t)=\sigma_{31}(t) \mathrm{e}^{-\mathrm{i} \omega t}, \rho_{m m}(t)=\sigma_{m m}(t), m=1,2,3,
$$


of the electron-hole excitation in such QWs can be reduced to very symmetric system

$$
\left\{\begin{array}{l}
\frac{\mathrm{d} \sigma_{11}}{\mathrm{~d} t}=-2 \Omega(t) \operatorname{Im}\left(\sigma_{s}\right) \\
\frac{\mathrm{d} \sigma_{s}}{\mathrm{~d} t}+\left(\gamma+\mathrm{i} \frac{\omega_{32}}{2}\right) \sigma_{s}=\mathrm{i} \frac{\Omega(t)}{2}\left[\frac{1}{2}\left(3 \sigma_{11}-1\right)-\rho_{32}\right] \\
\frac{\mathrm{d} \rho_{32}}{\mathrm{~d} t}+\left(\gamma_{32}+\mathrm{i} \omega_{32}\right) \rho_{32}=-\mathrm{i} \Omega(t) \sigma_{s}
\end{array}\right.
$$

Here $E(t)$ is the laser field amplitude and the following relations are used:

$$
\sigma_{31}=\sigma_{s}, \quad \sigma_{21}=-\sigma_{s}^{*}, \quad \sigma_{22}(t)=\sigma_{33}(t)=\frac{1}{2}\left[1-\sigma_{11}(t)\right]
$$

For the sake of simplicity, let us consider the case $\gamma=\gamma_{32}=0$ and a $\pi$-shaped laser pulse of duration $\tau_{\mathrm{p}}$. Then, for the ground-state population $\sigma_{11}(t)$ and the mid/far-infrared coherence $\rho_{32}(t) \equiv \rho_{32}^{\prime}(t)+\mathrm{i} \rho_{32}^{\prime \prime}(t)$ at the time interval $0<t<\tau_{\mathrm{p}}$ the following solution is valid:

$$
\begin{aligned}
\sigma_{11}(t) & =\frac{1}{2}+\frac{2}{3} \frac{\left(\Omega_{0}^{2}-\omega_{32}^{2}\right)^{2}}{\left(2 \Omega_{0}^{2}+\omega_{32}^{2}\right)^{2}}+\frac{2 \Omega_{0}^{4}}{\left(2 \Omega_{0}^{2}+\omega_{32}^{2}\right)^{2}} \cos \left(\sqrt{2 \Omega_{0}^{2}+\omega_{32}^{2}} t\right) \\
+ & \frac{4 \Omega_{0}^{2} \omega_{32}^{2}}{\left(2 \Omega_{0}^{2}+\omega_{32}^{2}\right)^{2}} \cos \left(\frac{1}{2} \sqrt{2 \Omega_{0}^{2}+\omega_{32}^{2} t}\right), \\
\rho_{32}^{\prime}(t) & =\frac{\Omega_{0}^{2}\left(\Omega_{0}^{2}+\omega_{32}^{2}\right)}{\left(2 \Omega_{0}^{2}+\omega_{32}^{2}\right)^{2}}\left[1-\cos \left(\sqrt{2 \Omega_{0}^{2}+\omega_{32}^{2}} t\right)\right] \\
- & \frac{2 \Omega_{0}^{2} \omega_{32}^{2}}{\left(2 \Omega_{0}^{2}+\omega_{32}^{2}\right)^{2}}\left[1-\cos \left(\frac{1}{2} \sqrt{2 \Omega_{0}^{2}+\omega_{32}^{2}} t\right)\right] \\
\rho_{32}^{\prime \prime}(t) & =\frac{\Omega_{0}^{2} \omega_{32}}{\left(2 \Omega_{0}^{2}+\omega_{32}^{2}\right)^{3 / 2}} \sin \left(\sqrt{2 \Omega_{0}^{2}+\omega_{32}^{2}} t\right) \\
- & \frac{2 \Omega_{0}^{2} \omega_{32}}{\left(2 \Omega_{0}^{2}+\omega_{32}^{2}\right)^{3 / 2}} \sin \left(\frac{1}{2} \sqrt{2 \Omega_{0}^{2}+\omega_{32}^{2}} t\right) .
\end{aligned}
$$

As one can see from Eqs. (5)-(7), if the duration of the pulse is equal to the integer number of periods $T=4 \pi / \sqrt{2 \Omega_{0}^{2}+\omega_{32}^{2}}$, one will get the system in the initial state with $\rho_{32}\left(\tau_{\mathrm{p}}\right)=\sigma_{22}\left(\tau_{\mathrm{p}}\right)=\sigma_{33}\left(\tau_{\mathrm{p}}\right)=0$, i.e., the coherence oscillations will stop as soon as the pump pulse ends. The results of numerical simulations with a finite coherence relaxation included $\left(\gamma=\gamma_{32}=10^{13} \mathrm{~s}^{-1}\right)$ are shown in Fig. 3 and match well the solution (5)-(7) for the short enough pump pulse $\left(\tau_{\mathrm{p}}<1 / \gamma, 1 / \gamma_{32}\right): \rho_{32} \approx 0$ and $\sigma_{22,33}<\sigma_{11}$ at $\tau>\tau_{\mathrm{p}}$ for the curve 2 .

An essential shortening of the generated mid/far-infrared pulse due to cut-off of the afterglow rings of the intersubband coherence $\rho_{32}$ is quite general effect that takes place for any other smoothly-shaped pump pulse. It was shown by numerical simulations, e.g., for the secant-shaped pump pulse $\Omega(t)=\Omega_{0} / \cosh \left[\left(t-t_{0}\right) / \tau_{\mathrm{p}}\right]$ and Gaussian pump pulse. In particular, it was demonstrated that the amplitude 

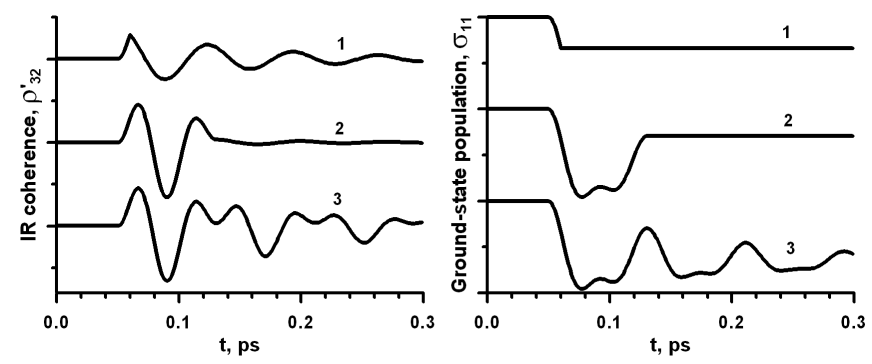

Fig. 3. Coherence $\rho_{32}^{\prime}(t)$ (left part) and population $\sigma_{11}(t)$ (right part) for the $\pi$-shaped optical excitation pulse. Parameters: $\omega_{32}=90 \times 10^{12} \mathrm{~s}^{-1}, \gamma_{32}=\gamma=10^{13} \mathrm{~s}^{-1}$. The amplitude of the pump pulse is fixed, $\Omega_{0} \equiv d E / \hbar=90 \times 10^{12} \mathrm{~s}^{-1}$, and its duration $\tau_{\mathrm{p}}=(10,80,300) \mathrm{fs}$.

of the mid/far-infrared coherence and the difference-frequency signal decreases sharply as soon as the width of the pump pulse spectrum becomes less than $\omega_{32}$.

\section{Estimation and conclusion}

For the estimates, let us take typical values of the parameters for GaAs-based heterostructures: $\omega_{32}=90 \times 10^{12} \mathrm{~s}^{-1}$ (i.e., $14.3 \mathrm{THz}$ or $\lambda_{\mathrm{IR}} \approx 21 \mu \mathrm{m}$ ), $d_{21} / e \sim d_{31} / e \sim 1 \mathrm{~nm}, d_{32} / e \sim 3 \mathrm{~nm}, \varepsilon \sim 12, m_{\mathrm{e}} \approx 0.06 m_{0}$ (electron mass in the conduction band in terms of the free-electron mass). For $N_{\mathrm{QW}}=100$ (i.e., a total thickness of the multiple QWs of order $1 \mu \mathrm{m}$ ) and the amplitude of the electric field in the optical pump pulse $E \approx 5 \times 10^{5} \mathrm{~V} \mathrm{~cm}^{-1}$, we find

$$
\max \left|E_{\mathrm{IR}}(t)\right| \sim \frac{4 \pi d_{32} 0.1 \omega_{32}}{c \varepsilon^{3 / 2}} N_{0} N_{\mathrm{QW}} \approx 100\left[\mathrm{~V} \mathrm{~cm}^{-1}\right]
$$

In terms of peak intensities, the latter means that one gets $13 \mathrm{~W} \mathrm{~cm}^{-2}$ of the mid/far-infrared radiation out of $300 \mathrm{MW} \mathrm{cm}^{-2}$ of the optical pump. The corresponding number densities of the optical and mid/far-infrared photons per unit area per pulse at $\tau_{\mathrm{p}} \approx 30$ fs are of order $4 \times 10^{13} \mathrm{~cm}^{-2}$ and $4 \times 10^{7} \mathrm{~cm}^{-2}$, respectively. There are different ways to increase the conversion efficiency, e.g., by using higher intensities of the optical pump pulses, optimized geometry, diffraction grading on top of the structure, more favourable, in-QW-plane polarization of the heavy-light hole transitions, other III-V semiconductor materials, etc.

The experiments outlined in the present paper are under way for the InGaAs/GaAs/InGaAsP and AlGaAs/GaAs multiple-QW heterostructures with the interband transitions in the range $0.91-0.94 \mu \mathrm{m}$ and $0.79-0.81 \mu \mathrm{m}$ wavelengths, respectively.

In conclusion, we present a simple model describing the generation of short mid/far-infrared pulses via the resonant nonlinear-wave mixing within the band width of the pump femtosecond pulse in the multiple-QW heterostructures. On the basis of the analytical solution for a $\pi$-shaped optical pump pulse, we found 
that one can expect the generation of the utmost short mid/far-infrared pulses with a few- or even single-cycle duration if the proper adjustment of the pump pulse parameters is done. The explicit formula for the mid/far-infrared electric field is derived, and its peak value is estimated as $100 \mathrm{~V} / \mathrm{cm}$ that is high enough for many applications.

\section{Acknowledgments}

This work was supported by awards from DARPA, ONR, the Research Corporation, the TAMU Telecommunication and Informatics Task Force Initiative, the Robert A. Welch Foundation (grants No. A-1261 and A-1218), and RFBR No. 02-02-17536, Sh-1744.2003.2.

\section{References}

[1] A. Rice, Y. Jin, X.F. Ma, Z.-C. Zang, D. Bliss, J. Larkin, M. Alexander, Appl. Phys. Lett. 64, 1324 (1994).

[2] N. Sarukura, H. Ohtake, S. Izumida, Z. Liu, J. Appl. Phys. 84, 654 (1998).

[3] J.-P. Likforman, M. Mehendale, D.M. Villeneuve, M. Joffre, P.B. Corkum, Opt. Lett. 26, 99 (2001).

[4] P. Gu, M. Tani, S. Kono, K. Sakai, X.-C. Zhang, Appl. Phys. Lett. 91, 5533 (2002).

[5] X.-C. Zhang, D.H. Auston, J. Appl. Phys. 71, 326 (1992).

[6] R. McLaughlin, Q. Chen, A. Corchia, C.M. Ciesla, D.D. Arnone, X.-C. Zhang, G.A.C. Jones, E.H. Linfield, M. Pepper, J. Mod. Opt. 47, 1847 (2000).

[7] J.T. Darrow, B.B. Hu, X.-C. Zhang, D.H. Auston, Opt. Lett. 15, 323 (1990).

[8] G. Zhao, R.N. van der Schouten, N. Valk, W.Th. Wenckebachand, P.C.M. Planken, Rev. Sci. Instrum. 73, 1715 (2002).

[9] M.C. Beard, G.M. Turnerand, C.A. Schmuttenmaer, J. Appl. Phys. 90, 5915 (2001).

[10] H.G. Roskos, M.C. Nuss, J. Shah, K. Leo, D.A.B. Miller, A.M. Fox, S. Schmitt-Rink, K. Kohler, Phys. Rev. Lett. 68, 2216 (1992).

[11] P.C.M. Planken, M.C. Nuss, I. Brener, K.W. Goosen, M.S.C. Luo, S.L. Chuang, L. Pfeiffer, Phys. Rev. Lett. 69, 3800 (1992).

[12] M.S.C. Luo, S.L. Chuang, P.C.M. Planken, I. Brener, M.C. Nuss, Phys. Rev. B 48, 11043 (1993).

[13] M.S.C. Luo, S.L. Chuang, P.C.M. Planken, I. Brener, H.G. Roskos, M.C. Nuss, IEEE J. Quant. Electron. 30, 1478 (1994).

[14] P.G. Huggard, C.J. Shaw, S.R. Andrews, J.A. Cluff, R. Grey, Phys. Rev. Lett. 84, 1023 (2000).

[15] C. Waschke, H.G. Roskos, R. Schwedler, K. Leo, H. Kurz, K. Kohler, Phys. Rev. Lett. 70, 3319 (1993). 
[16] R. Martini, G. Klose, H.G. Roskos, H. Kurz, H.T. Grahn, R. Hey, Phys. Rev. B 54, 14325 (1996).

[17] K. Jin, M. Odnoblyudov, Y. Shimada, K. Hirakawa, K.A. Chao, Phys. Rev. B 68, 153315 (2003).

[18] A.A. Belyanin, F. Capasso, V.V. Kocharovsky, Vl.V. Kocharovsky, M.O. Scully, Phys. Rev. A 63, 053803 (2001).

[19] A.A. Belyanin, V.V. Kocharovsky, Vl.V. Kocharovsky, M.O. Scully, Phys. Rev. A 65, 053824 (2002).

[20] A.A. Belyanin, D. Deppe, V.V. Kocharovsky, Vl.V. Kocharovsky, D.S. Pestov, M.O. Scully, Physics-Uspekhi 46, 986 (2003).

[21] A. Bonvalet, J. Nagle, V. Berger, A. Migus, J.-L. Martin, M. Joffre, Phys. Rev. Lett. 76, 4392 (1996).

[22] Y.-C. Chang, R.B. James, Phys. Rev. B 39, 12672 (1995). 